\title{
Diseño metodológico
}

\section{de la Encuesta Nacional sobre Violencia contra las Mujeres en México}

\author{
Gustavo Olaiz, M en C,(1) Aurora Franco, Lic en Tr Soc,(1) Oswaldo Palma, Act, (1) Carlos Echarri, Dr en Demografía,(2) \\ Rosario Valdez, M en Antrop, ${ }^{(1)}$ Cristina Herrera, M en C. (I)
}

Olaiz G, Franco A, Palma O, Echarri C, Valdez R, Herrera C. Diseño metodológico de la Encuesta Nacional sobre Violencia contra las Mujeres en México. Salud Publica Mex 2006;48 supl 2:S328-S335.

\section{Resumen}

Objetivo. Describir la metodología utilizada en la Encuesta Nacional sobre Violencia contra las Mujeres 2003 (ENVIM 2003) en México, junto con el diseño de investigación, la estimación y la selección de muestras, la definición de variables, los instrumentos de recolección, el diseño operativo para su instrumentación y los procedimientos de análisis. Material y métodos. En la parte cuantitativa se recurrió a un diseño transversal en dos etapas. En el componente cualitativo se realizaron entrevistas en profundidad y observación participante en unidades médicas. Resultados. Se obtuvo un total de 26240 entrevistas, aplicadas a usuarias de los servicios de salud; y 2636 cuestionarios correspondientes a proveedores, que abarcaron los 32 estados de la República. En el estudio cualitativo se llevó a cabo un total de 26 entrevistas de profundidad a usuarias y 60 entrevistas de profundidad a prestadores de servicios de salud en los estados de Quintana Roo, Coahuila y el Distrito Federal.

Palabras clave: violencia de pareja; prestadores de servicios de salud; diseño muestral; México
Olaiz G, Franco A, Palma O, Echarri C, Valdez R, Herrera C. Methodological Design for the National Survey Violence Against Women in Mexico.

Salud Publica Mex 2006;48 suppl 2:S328-S335.

\section{Abstract}

Objective. To describe the methodology, the research designs used, the estimation and sample selection, variable definitions, collection instruments, and operative design and analytical procedures for the National Survey Violence Against Women in Mexico. Material and Methods. A complex (two-step) cross-sectional study was designed and the qualitative design was carried out using in-depth interviews and participant observation in health care units. Results. We obtained for the quantitative study a total of 26240 interviews in women users of health services and 2636 questionnaires for health workers; the survey is representative of the 32 Mexican states. For the qualitative study 26 in-depth interviews were conducted with female users and 60 interviews with health workers in the States of Quintana Roo, Coahuila and the Federal District.

Key words: family violence; health workers; sampling design; Mexico
L a violencia de pareja es un problema social que $\triangle$ debe ser reconocido por toda la población. Ocuparse de ella es darle la debida importancia a uno de los problemas de salud pública que más se ha mantenido oculto a lo largo de la historia. De aquí la importancia de romper con la idea de que es un problema "privado" y hacer públicas estas situaciones que dañan la salud de millones de personas. La violencia de pareja constituye un delito que afecta y deteriora los aspectos más valiosos del ser humano, y puede llegar a costarle la vida. ${ }^{1,2}$ Desde la perspectiva de salud pública, la violencia de pareja es un problema que ocasiona daño psi-

(I) Instituto Nacional de Salud Pública, México.

(2) El Colegio de México.

Fecha de recibido: 26 de agosto de 2004 - Fecha de aprobado: 25 de mayo de 2005 Solicitud de sobretiros: Lic. Aurora Franco, $7^{\text {a }}$ Cerrada de Fray Pedro de Gante No. 50, Col. Sección XVI, Delg. Tlalpan, México, D.F. Correo electrónico: afranco@insp.mx 
cológico además del daño físico, así como discapacidad, secuelas y disminución de la calidad de vida., ${ }^{2,3}$

La demanda femenina de servicios médicos para la atención de lesiones o secuelas físicas, ocasionadas por violencia de la pareja masculina, fue mínima en estas mujeres, por lo que obtener una estimación sobre la importancia de este problema de salud a partir de los registros de morbilidad médicos resulta insuficiente y subestima la magnitud del problema para los servicios de salud. ${ }^{3}$ Por otra parte, no existen estudios que permitan estimar la prevalencia de este problema de salud en el ámbito nacional.

Entre 2002 y 2003, el Instituto Nacional de Salud Pública, el Programa de Salud de la Mujer de la Secretaría de Salud e investigadores invitados formaron el grupo de trabajo de investigación sobre violencia contra la mujer. Este diseñó el estudio encaminado a mostrar la perspectiva general sobre la violencia contra la mujer, tanto en usuarias de los servicios de salud como en proveedores. Para este fin se utilizaron metodologías de tipo cuantitativo y cualitativo. El grupo de trabajo ha intentado determinar la prevalencia de este problema en la población de usuarias de servicios de salud dentro del ámbito nacional. Para la medición de la violencia se consideraron múltiples perspectivas; se incluyeron tanto metodologías cuantitativas como cualitativas que dieran respuesta a los siguientes objetivos: a) estimar la prevalencia de los diferentes tipos de violencia en la población analizada; b) identificar cualitativamente los factores que dificultan una adecuada respuesta al problema de la violencia de pareja; c) indagar en la perspectiva de las mujeres acerca de la manera en que entienden la violencia de su pareja hacia ellas; d) conocer las actitudes, los cursos de acción y las barreras institucionales que enfrentan los prestadores de servicios al tratar este tipo de problema, y e) aportar elementos para el diseño de intervenciones que promuevan una atención eficaz y adecuada a este problema de salud.

\section{Diseño del estudio}

El diseño de investigación sobre la violencia de pareja en México implicó abordar una variedad de aspectos (sociales, económicos, culturales y de salud) relacionados con la percepción y aceptación del problema de la violencia. Para su estudio se diseñaron, utilizaron y conjugaron estrategias metodológicas de tipo cuantitativo y cualitativo.

En lo referente al ámbito cuantitativo, y con el propósito de recabar evidencia empírica confiable sobre el problema, se recurrió al diseño transversal, el cual ofrece la ventaja de permitir una rápida exploración de la po- blación sobre los factores de interés. ${ }^{4}$ Entre las bondades de este tipo de diseño se encuentran el facilitar la incorporación de diversos módulos de estudio, y su cobertura puede ser tan amplia o específica como se requiera; además, este tipo de diseño ha sido utilizado para la evaluación de políticas públicas y la planeación en salud. Dentro de esta metodología se incluyó a los 32 estados de la República y se seleccionaron 10 unidades médicas en cada uno de ellos, con lo cual se abarcó a las siguientes instituciones: Instituto de Seguridad y Servicios Sociales para los Trabajadores del Estado (ISSSTE), Instituto Mexicano del Seguro Social (IMSS) y Secretaría de Salud (SSA). Se captó a mujeres usuarias y a proveedores de servicios de salud. El estudio orientado a proveedores de salud se diseñó para identificar la respuesta institucional relacionada con la demanda y atención del problema de violencia de pareja, lo cual permite conocer la respuesta de los prestadores de servicios de salud ante este problema. En síntesis, se desarrolló una encuesta nacional para conocer la magnitud de la violencia de pareja en mujeres usuarias de los servicios públicos de salud, llamada Encuesta Nacional sobre Violencia contra las Mujeres 2003 (ENVIM 2003), la cual incluyó un instrumento de autoaplicación para los prestadores de servicios de salud.

Con el estudio cualitativo se exploraron tanto las perspectivas de las mujeres usuarias como de los prestadores de servicios de salud sobre la violencia contra las mujeres en tres entidades federativas, que fueron seleccionadas por su alta prevalencia de violencia de pareja contra la mujer (Quintana Roo, Coahuila y el Distrito Federal). Se utilizaron diversas técnicas cualitativas, como la entrevista en profundidad, la entrevista narrativa y la observación participante en unidades de salud de las entidades seleccionadas, con la ayuda de guías temáticas. La combinación de información cuantitativa y cualitativa facilitó el análisis integral sobre la problemática de salud de la violencia de pareja.

A continuación se presenta una descripción detallada de cada uno de los estudios referidos.

Métodos cuantitativos para el levantamiento de la información

\section{Objetivo}

El objetivo principal de la encuesta fue realizar un estudio en el ámbito nacional sobre la violencia de pareja en mujeres que demandan atención en los servicios médicos de las instituciones públicas de primer y segundo niveles en México, para estimar la prevalencia de la violencia de pareja en mujeres, así como identificar el tipo de violencia a través de la cuantificación de frecuencia y severidad. 
Unidad de observación

Se definió como sujetos de estudio a las mujeres de 15 años y más, usuarias de los servicios públicos de salud de primer y segundo niveles de atención que solicitaran el servicio médico, independientemente del problema de salud, y que fueran seleccionadas de forma sistemática.

\section{Diseño operativo}

La encuesta se realizó durante los meses de noviembre y diciembre de 2002 y de enero a marzo de 2003, en las unidades médicas de primer y segundo niveles de la SSA, del IMSS e ISSSTE, los cuales representaron a los 32 estados de la República mexicana.

Para el levantamiento de la encuesta se desarrollaron las siguientes actividades:

1. Sensibilización de autoridades estatales.

Antes de iniciar el trabajo de campo en cada estado se organizó una reunión presidida por autoridades de la SSA, IMSS, ISSSTE, Sistema Nacional para el Desarrollo Integral de la Familia (DIF) y organizaciones no gubernamentales que trabajan en el área de la violencia contra la mujer. En dicha reunión se presentaron los objetivos y el desarrollo del proyecto.

2. Reclutamiento del personal.

Las entrevistadoras fueron reclutadas a través de convocatorias realizadas en facultades y escuelas de enfermería, trabajo social, psicología y sociología.

3. Selección del personal.

Era necesario garantizar la neutralidad durante la entrevista y que el personal seleccionado pudiera manejar la problemática de una mujer, por lo que a todas las aspirantes a entrevistadora y supervisora se les evaluó mediante una entrevista personal para identificar su sensibilidad ante el tema de violencia y su capacidad para manejarlo.

4. Capacitación.

La capacitación tuvo una duración de tres meses, durante los cuales se llevaron a cabo diversas pruebas de campo y ajustes al cuestionario, y se compuso de dos etapas. La primera consistió en el manejo del cuestionario y de las técnicas para aplicar la entrevista. Se realizaron socio dramas, prácticas de campo y evaluaciones de los temas explorados en el cuestionario. En la segunda etapa se capacitó al personal en técnicas para el manejo de mujeres en situación de violencia y se revisaron las definiciones de los diferentes tipos de violencia. Se realizaron dinámicas y talleres de integración grupal.
También hubo sesiones personalizadas con las entrevistadoras que lo requerían.

5. Estrategia de levantamiento.

Para el levantamiento de la encuesta se implementó un operativo con 100 entrevistadoras, 16 supervisoras y 10 coordinadoras divididas en 10 equipos. El trabajo de campo se distribuyó en cinco regiones, cada una de las cuales fue trabajada por dos equipos y finalmente se integró con el levantamiento de seis estados. La programación del trabajo de campo permitió dejar para las últimas etapas del levantamiento al Estado de México y al Distrito Federal, con lo que se redujeron las dificultades operativas. Selección operativa de la informante. Para seleccionar a las usuarias se solicitó previamente al director de la unidad médica correspondiente una relación de las consultas que se dieron a mujeres durante la semana previa al levantamiento. Se tomó en cuenta el día, el servicio solicitado y el turno. Se consideró esta población como base para la determinación de la fracción muestral. Cabe mencionar que con la intención de no subestimar o sobreestimar los casos de violencia y evitar el sesgo de estacionalidad, las entrevistadoras permanecieron en las unidades médicas durante una semana laboral. Las mujeres entrevistadas fueron captadas en los servicios de primer y segundo niveles de atención en urgencias, medicina interna y ginecobstetricia.

Privacidad. Antes de iniciar la entrevista se entregó a cada entrevistada la carta de consentimiento informado, con la cual se garantizó la confidencialidad de la información. Con el fin de lograr un ambiente de confianza para las entrevistadas en cada unidad médica se solicitó un espacio cerrado para realizar las entrevistas.

Manejo de estrés. Se brindó apoyo a las mujeres que durante la entrevista presentaron angustia o alguna crisis emocional. Además, si ella lo solicitaba, se le transfería a alguna de las instituciones estatales de apoyo a las mujeres violentadas.

Retroalimentación. Se diseñó un tríptico en el que se explica el ciclo de violencia, y un directorio, para cada estado, de tres o cuatro instituciones que atienden a mujeres en situación de violencia. La selección de estas instituciones se acordó conjuntamente con las autoridades estatales encargadas del tema (SSA, IMSS, ISSSTE, DIF y organizaciones de apoyo a la mujer).

6. Supervisión.

Se preguntó a 10\% de las entrevistadas si habían recibido el tríptico, su opinión al respecto y si querían hacer alguna observación acerca del desem- 
peño de la entrevistadora. Para la supervisión directa fue necesario que tanto la entrevistadora como la supervisora se identificaran al iniciar la entrevista, a fin de que ésta pudiera desarrollarse en presencia de ambas. El total de las entrevistas fue revisado en campo por las supervisoras y $30 \%$ por parte de las coordinadoras. La selección aleatoria de las entrevistadas fue verificada mediante la observación directa del trabajo en campo.

\section{Instrumento para recolectar información en usuarias}

El cuestionario de la ENVIM estuvo integrado por 17 secciones, en las cuales se exploró la siguiente información: identificación de hogares, datos generales, identificación de la demanda del servicio, características de la vivienda y del hogar, características demográficas de la encuestada, salud general y personal de la encuestada, alcoholismo en la mujer, uniones de pareja, características demográficas del esposo, compañero o última pareja, alcoholismo del compañero, percepciones de la encuestada hacia los roles de género, relaciones familiares y de pareja, caracterización del maltrato, evaluación de la respuesta del personal de salud ante la violencia de pareja y toma de decisiones familiares.

Proveedores de servicios de salud

Selección de los proveedores de salud. Para la autoaplicación del cuestionario se seleccionaron 10 unidades médicas en cada entidad federativa. Se abarcaron el primer y segundo niveles de atención de los servicios de urgencias, medicina interna y ginecología; en estas unidades se seleccionó a médicas, enfermeras, trabajadoras sociales y psicólogas, para la autoaplicación del cuestionario de proveedores de salud. La selección se realizó de manera aleatoria y con base en la cobertura de los prestadores de servicios existentes en cada unidad médica.

Instrumento para recolectar información en proveedores. Se aplicó un cuestionario al personal de salud, el cual contiene: a) Identificación geográfica, en que se registró la ubicación de la unidad médica, el tipo de institución a la cual pertenecía, así como el tipo de comunidad donde estaba ubicada. b) Identificación, en esta sección se registraron los datos generales de cada participante: edad, sexo, estado civil, profesión, grado máximo de estudios y categoría ocupada dentro de la institución. c) Conocimiento sobre violencia, en esta sección se investigó sobre el conocimiento y la capacitación recibida por parte del personal de salud en relación al diagnóstico y manejo de la violencia de pareja, así como su grado de interés en el problema y su posible participación en relaciones violentas, ya sea como agredido/a o agresor/a.

\section{Diseño muestral}

El diseño muestral de la ENVIM fue realizado con el fin de proporcionar estimaciones tanto en el ámbito nacional como en las entidades federativas. Las instituciones públicas de salud (IMSS, ISSSTE y SSA) conformaron los estratos de la encuesta. Entre estos estratos la muestra fue asignada de manera proporcional al número de usuarios de la institución en el estado según los hallazgos de la Encuesta Nacional de Salud 2000. Por razones de tipo operativo y de costos, el número de unidades médicas visitadas por estado se fijó en 10.

Con base en la revisión de la literatura y la consulta a expertos, se concluyó que la prevalencia de violencia actual sería superior a 10\%. Esta proporción fue considerada para el cálculo del tamaño de muestra por entidad federativa. Otros parámetros fueron: tasa de no respuesta máxima por entidad de $15 \%$, error relativo máximo $r=0.2$, confianza del $95 \%\left(Z_{\alpha / 2}^{2}=1.96\right)$ y efecto de diseño deff $=1.7 .{ }^{5}$ De acuerdo con la siguiente fórmula:

$$
n=\frac{Z_{\alpha / 2}^{2}(1-P) d e f f}{r^{2} P T R}
$$

se determinó que se requería entrevistar a por lo menos 820 mujeres por estado.

Este estudió se basó en una muestra probabilística compuesta por dos etapas. ${ }^{6}$ En la primera se seleccionaron unidades médicas con probabilidad proporcional al número de consultorios de la unidad, de entre un listado de las unidades posibles a seleccionar proporcionado por la Secretaría de Salud para cada una de las instituciones participantes. La segunda etapa consistió en la selección de mujeres mayores de 14 años que acudieran a la unidad médica con la finalidad de recibir atención preventiva o curativa. La selección de las mujeres se realizó a través de un muestreo sistemático con arranque aleatorio, ${ }^{7}$ ya que se desconocía el número de mujeres que acudirían a los servicios de salud durante el desarrollo del operativo en la unidad. Con la intención de eliminar la posibilidad de sesgos de selección asociados a la variación de la demanda de los servicios de salud, la metodología requería que el operativo de campo se implementara durante todos los días laborables de una semana habitual en la unidad. Se determinó una fracción de muestreo tomando como tamaño esperado de la población el percentil 85 de las consultas o intervenciones a mu- 
jeres mayores de 14 años en esa unidad. Es decir, la fracción de muestreo que se utilizó fue:

$$
f=0.85 \frac{m_{k} \cdot d}{82}
$$

donde $m^{k}$ representa el número de mujeres adultas que demandaron servicios en la unidad visitada el $k$-ésimo día de la semana previa y $d$ representa el número de días laborables en la unidad durante la semana previa.

\section{Medición de variables}

Se utilizaron diversas escalas para medir algunas de las variables de interés:

\section{Medición de violencia}

Para la medición de la violencia de pareja se utilizó una escala que constó de 27 reactivos obtenidos del Index of Spouse Abuse (ISA) y del Severity of Violence Against Women Scale (SVAWS). Además, se incluyeron otros dos reactivos para explorar la violencia económica. Se midió la frecuencia de las acciones violentas en los últimos 12 meses ( $1=$ nunca, $2=$ alguna vez, $3=$ varias veces y $4=$ muchas veces). La escala incluye las siguientes dimensiones:

Violencia fisica: empujones, golpes, heridas de arma de fuego o punzocortante.

Violencia emocional: intimidación, humillaciones, amenaza de violencia física.

Violencia sexual: forzar física o emocionalmente a la mujer a la relación sexual.

Violencia económica: ejercicio del poder a través del control del dinero.

\section{Medición de salud personal}

Para medir el malestar emocional se utilizó la Escala de Salud Personal (ESP) desarrollada en Nicaragua por un grupo de profesionales de la salud mental. La ESP fue originalmente diseñada usando como base un cuestionario de autorreporte diseñado y publicado por Penayo y col. ${ }^{5}$ y Caldera y col. ${ }^{8}$ La ESP integra un total de 10 reactivos, los cuales hacen referencia a situaciones que pueden provocar malestar emocional y se miden utilizando las siguientes opciones $(0=$ raramente, $1=$ algunas veces y $2=$ casi siempre) que indican la frecuencia con que se manifestaron los síntomas descritos en cada reactivo durante el último mes. En el presente estudio se incorporó una opción de respuesta adicional para lograr mayor precisión: $1=$ nunca, $2=$ pocas veces, $3=$ varias veces y $4=$ casi siempre. Seis de las 10 preguntas de la escala se refieren a problemas somáticos y trastornos del afecto tales como insomnio, nerviosismo, tristeza y sobresalto. Tres se refieren a problemas funcionales: dificultades en el trabajo, la familia o en las relaciones sociales. La última pregunta es una autoevaluación global: " ¿Ha sentido que tiene problemas emocionales que requieran atención o ayuda profesional?"

Medición de estrato socioeconómico

La definición de los estratos socioeconómicos se basó en la combinación de tres características de los hogares: la escolaridad, la actividad de sus miembros y la cantidad de activos o equipos electrodomésticos existentes en la vivienda.

La primera dimensión, la escolaridad, fue calculada mediante el promedio de escolaridad de los miembros del hogar. Este indicador señala tanto la posesión de habilidades brindadas por el sistema educativo formal para el caso de aquellos que ya han terminado sus estudios, como las inversiones que hacen los hogares para aquellos de sus integrantes que se encuentran aún estudiando. Para poder tomar en cuenta las experiencias de todos los miembros del hogar, en el contexto de la expansión de la oferta educativa que ha existido en el país, se consideró de manera individual el número de años aprobados en la escuela con relación a un estándar. Para la construcción de este estándar se recurrió a la información del Conteo de Población de 1995, las Encuestas Nacionales de la Dinámica Demográfica (ENADID) de 1992 y 1997, y los Censos de 1990 y 2000, todos levantados por el Instituto Nacional de Estadística, Geografía e Informática (INEGI). Se calculó el promedio de años de escolaridad que se esperaba por sexo y generación. La escolaridad relativa se obtuvo restando el número de años estudiados por cada persona a la escolaridad estándar o esperada y dividiendo el resultado entre ese mismo estándar. Posteriormente se calculó el promedio para todos los miembros del hogar y se dividió en cuatro categorías:

1. Muy baja: cuando el valor obtenido en el promedio fue de hasta -0.25 .

2. Baja: para los valores de entre -0.26 y 0.1 .

3. Media: incluye los valores comprendidos entre 0.11 y 0.5 .

4. Alta: para valores por arriba de 0.5 veces.

La segunda dimensión que integra el índice es la ocupación. Para medirla se ordenaron las diferentes ocupaciones según su remuneración, de acuerdo con los datos de la Encuesta Nacional de Ingreso y Gasto en Hogares (ENIGH) de 1996, y para cada hogar se eligió la ocupación de la mujer o de su cónyuge asociada con 
un mayor ingreso. Este indicador da un acercamiento a las capacidades de generación de ingresos de los hogares mediante su inserción en el mercado laboral.

La tercera dimensión se refiere al entorno inmediato que caracteriza las condiciones de vida. El indicador obtenido para esta dimensión es un índice relativo de posesión de bienes; es decir, para cada hogar se dividió el número de bienes con los que contaba (teléfono celular, DVD, refrigerador, lavadora, estufa de gas, calentador de agua y automóvil o camioneta), así como la ausencia de hacinamiento.* La cantidad obtenida se dividió entre el número de variables utilizadas. El índice se dividió en cuartiles.

Finalmente, se calculó una combinación lineal de estas tres dimensiones, las 192 categorías resultantes se ordenaron de acuerdo con el ingreso per cápita del hogar obtenido en la Encuesta Nacional de Dinámica Demográfica (ENADID) de 1997 y se dividieron en cuatro categorías, las cuales conforman los estratos. Cabe aclarar que esta metodología es una adaptación de la que fue diseñada para el proyecto Diagnóstico de la salud reproductiva en el México de los noventa, llevado a cabo por el Programa Salud Reproductiva y Sociedad de El Colegio de México, lo que permite aprovechar la experiencia obtenida en su aplicación a las encuestas nacionales de salud reproductiva, y facilita la comparanza de los estratos socioeconómicos obtenidos con los de otras fuentes para validar y contextualizar los resultados.

\section{Resultados}

Luego de considerar a los 32 estados de la República, se obtuvo un total de 26240 entrevistas a usuarias de los servicios de salud. En promedio correspondieron a cada estado 820 cuestionarios y respecto a los proveedores de salud se obtuvo un total de 2636 entrevistas. En todos los casos la ausencia de respuesta se mantuvo dentro de lo anticipado en el diseño.

Cabe mencionar que los resultados obtenidos incluyeron solamente a la población que demandó atención en los servicios médicos de las instituciones seleccionadas.

\section{Métodos cualitativos para el levantamiento de la información}

Objetivo

El objetivo general fue explorar a profundidad los factores personales, sociales, culturales e institucionales

\footnotetext{
* Se considera hacinamiento cuando existen 2.5 habitantes o más por dormitorio.
}

que afectan la percepción, experiencia y búsqueda de solución al problema por parte de los principales protagonistas de la interacción en los servicios: los prestadores de servicios de salud y las mujeres que sufren o han sufrido violencia de pareja.

\section{Aspectos teórico-metodológicos}

Para lograr los objetivos planteados se buscó construir el significado que los mismos actores sociales asignan a este fenómeno de violencia en pareja; es decir, la forma en que entienden la violencia contra las mujeres, a qué atribuyen el problema, sus orientaciones y estrategias de acción para resolverlo. Se recabó información sobre el conocimiento del problema, así como sobre la denominada "experiencia ateórica", de carácter prerreflexivo, ${ }^{9}$ constituida por conocimientos que orientan la acción pero no han sido objeto de una reflexión consciente por parte de los agentes sociales.

El marco conceptual que sustenta esta estrategia metodológica cualitativa enfoca la violencia de pareja desde una perspectiva social y no meramente individual o de corte psicopatológico. Desde este enfoque, la forma en que los afectados viven y afrontan el problema es, por una parte, producto de un proceso social de acción y comunicación, el cual es responsable de las formas de comprensión del fenómeno en general y, por la otra, depende de la historia del individuo. Por lo que de esta concepción se deriva el supuesto de que los relatos obtenidos por los individuos reflejan el conocimiento del fenómeno de la violencia como un producto del intercambio social del que provienen, siendo clave para este estudio el que se produce entre los géneros y las normas sociales que lo regulan.

\section{Técnicas de recolección de datos}

Para recabar información sobre esta problemática se utilizaron diferentes herramientas cualitativas de recolección de datos:

Observación directa en los servicios de salud y diario de campo

El espacio de observación fue el de la interacción que se produce en los servicios de salud, entre las mujeres que solicitan atención y quienes la brindan. Si bien se observó la interacción con el personal administrativo (trámites requeridos, obstáculos burocráticos, trato a las usuarias por parte del personal, etc.), el énfasis estuvo puesto en las actitudes y prácticas de los proveedores de servicios, incluyendo enfermeros/as, médicos/as, psicólogos/as y trabajadores/as sociales, con el fin de observar algunos indicadores de calidad de atención. 


\section{Entrevistas en profundidad con prestadores de servicios}

En este caso la prioridad fue observar la distorsión o concordancia entre algunas creencias de los prestadores (de género y violencia, del perfil socioeconómico y cultural percibido de las víctimas, etc.) y sus prácticas frente a la violencia, sobre la base de su obligación de reportar y notificar casos, según la norma oficial ${ }^{10}$ y el hecho de que la violencia sea un delito. Para ello, se diseñó una guía de entrevista abierta que exploraba entre otros temas: a) adhesión a diferentes concepciones sobre el género, la familia y la violencia de pareja; b) actitudes frente a las mujeres que padecen violencia de pareja; c) sensibilidad ante el tema y, en consecuencia, calidad y calidez en la atención brindada; d) prácticas habituales frente al problema; e) conocimiento y aplicación de la norma oficial y motivos del conocimiento o desconocimiento y/o aplicación o no aplicación de la misma; f) resistencias para tratar el problema; g) coordinación y/o discrepancias entre el personal de salud (enfermeros/as, médico/as, trabajadores/as sociales, psicólogos/as e informantes clave); y h) disposición para atestiguar ante un proceso legal por violencia.

Entrevistas narrativas con mujeres víctimas de violencia de pareja

En este caso el propósito fue averiguar cómo entienden estas mujeres la violencia de su pareja contra ellas, a qué atribuyen el problema y qué estrategias implementan para su solución. Se buscó que las mismas mujeres organizaran, a partir de una pregunta estímulo, la narración de su experiencia de violencia de acuerdo con sus propios criterios. Se preparó un cuestionario con preguntas abiertas para guiar la conversación hacia aspectos que no se hubieran revelado espontáneamente durante la narración de la entrevistada. Entre estos aspectos estaban los siguientes: a) formas de percibir y significar la violencia de pareja (aspectos verbales y no verbales); b) relación de pareja y familia; c) vivencias personales en relación con el tema, historia y último episodio; d) redes de apoyo familiares y sociales; e) tolerancia a diferentes tipos de violencia; f) violencia durante el embarazo; g) proceso de búsqueda de atención médica y legal; h) satisfacción por la atención recibida; e i) necesidades y demandas insatisfechas, tipo de profesional que prefieren que trate este tema en los servicios de salud.

\section{Selección de los casos}

El trabajo de campo fue realizado entre mayo y agosto de 2003, en tres entidades con alta prevalencia de vio- lencia de pareja (Quintana Roo, Coahuila y el Distrito Federal) ${ }_{11}^{11}$ con la intención de replicarlo durante una segunda etapa, en dos estados con baja prevalencia (Aguascalientes y Morelos).

El muestreo fue realizado en el marco del análisis generativo propuesto por la teoría fundamentada en investigación cualitativa, ${ }_{12}^{12}$ la cual consiste básicamente en un proceso por el que se van elaborando hipótesis de trabajo para someterlas a su constatación mediante el estudio comparativo de casos. El llamado muestreo teórico $^{13}$ responde a los requerimientos de este tipo de análisis. Los casos se fueron seleccionando durante el mismo proceso en virtud de su valor informativo para la comprensión del fenómeno de manera general, y no por sus particularidades.

En el caso de las mujeres, se buscó una variación máxima de casos para evaluar las estrategias de acción y los recursos de los que se dispone para solucionar el problema. Por ello se buscó a mujeres que pertenecieran a generaciones diversas y que tuvieran distintas experiencias con la violencia: un grupo de ellas había logrado salir del problema, mientras que el otro aún se encontraba inmerso en una relación violenta. Se realizaron en total 26 entrevistas narrativas con mujeres víctimas de violencia, reclutadas en diferentes instituciones* en las tres entidades federativas mencionadas. Se buscó, asimismo, que las informantes correspondieran al perfil socioeconómico de usuarias de los servicios públicos de salud.

En el caso de los prestadores, se buscó obtener variación de acuerdo con su formación profesional, la institución y el tipo de servicios en los que desarrollaban su actividad. Se realizaron en total 60 entrevistas en profundidad con prestadores de las tres instituciones del sector (IMSS, ISSSTE y la SSA) en las tres entidades federativas. Estas se distribuyeron del siguiente modo: 16 médicos, 14 enfermeros, 11 psicólogos y 19 trabajadores sociales.

\section{Reclutamiento de los informantes y aspectos éticos del estudio}

El trabajo de campo fue realizado por un equipo de cuatro investigadores, conformado por dos psicólogos (uno de ellos especialista en violencia), una socióloga y una psicóloga social, con el objeto de responder a la necesidad de contar con personas aptas para contener alguna situación de crisis que pudiera presentarse en las usuarias víctimas de violencia. Todas las entrevistas, tanto

\footnotetext{
* Estas fueron: algunos servicios de salud del IMSS y de la SSA (entre ellos el centro especializado Sí Mujer en Coahuila), la Clínica de Atención a la Violencia Doméstica del ILEF, el Centro de Atención a la Violencia de pareja del DIF, y una ONG dedicada a atender a mujeres víctimas de violencia (CIAM, en Quintana Roo).
}

salud pública de méxico / vol.48, suplemento 2 de 2006 
las de las mujeres maltratadas como las de los prestadores de salud, se llevaron a cabo luego de la firma de una carta de consentimiento informado, en la que se explicaban los objetivos del estudio y se garantizaba la confidencialidad, y se realizaron en espacios privados como cubículos, oficinas, consultorios o domicilios particulares, asegurando de esta manera la privacidad.

En el caso de las mujeres, se les contactó a través de informantes clave, como trabajadoras sociales en los servicios de salud, personal del DIF y de otros centros de atención a víctimas de violencia, tanto gubernamentales como no gubernamentales y, profesionales de la salud mental que laboran en clínicas de atención a la violencia. Todas las mujeres mostraron total disposición a colaborar en el estudio y firmaron la carta de consentimiento informado correspondiente. Las entrevistas con las mujeres duraron en promedio una hora y media.

En el caso de los prestadores, con previa autorización de las instituciones correspondientes, se acudía a los servicios de salud y se establecía contacto con su director/a, a quien se explicaban los objetivos del estudio. Éste/a reclutaba a los prestadores que cubrieran el perfil solicitado por los investigadores y que en ese momento se encontraran disponibles y dispuestos a colaborar con el estudio. A estos mismos prestadores se les daba una nueva explicación detallada de los objetivos del estudio y se les invitaba a participar, previa firma de su consentimiento. En este caso, y salvo excepciones, también encontramos disposición a colaborar por parte de los/as entrevistados/as. Estas entrevistas fueron de menor duración que las de las mujeres, debido a que eran más dirigidas y a que se entrevistó a los prestadores en sus centros y horarios de trabajo. En promedio estas entrevistas duraron 40 minutos.

\section{Referencias}

I. Campero-Cuenca L. Educación y salud de la mujer: Reflexiones desde una perspectiva de género. Salud Pública Mex 1996;38:217-222.

2. Ramírez-Rodríguez JC, Uribe-Vázquez G. Mujer y violencia: Un hecho cotidiano. Salud Pública Mex 1993;35: I48-160.

3. Hijar M, López MV. La violencia y sus repercusiones en la salud: Reflexiones teóricas y magnitud del problema en México. Salud Pública Méx 1997;39:565-572.

4. Rothman KJ, Greenland S. Modern epidemiology. 2a edición. Filadelfia: Lippincott-Raven, 1998.

5. Penayo U, Caldera T, Jacobsson L. Prevalencia de trastornos mentales en adultos de Subtiava, León, Nicaragua. Boletín de la Oficina

Panamericana de Salud 1992; I 3:137-I 49.

6. Lohr S. Muestreo, diseño y análisis. México: Editorial Thomson, 2000.

7. Cochran WG. Técnicas de muestreo. México: Editorial Continental, 1980.

8. Caldera JT, Kullgren G, Penayo U, Jacobsson L. Is treatment in groups

a useful alternative for psychiatry in low-income countries? An

evaluation of a psychiatric outpatient unit in Nicaragua. Acta Psiquitr Scand 1995;92:386-391.

9. Mannheim K. Strukturen des Denkens. Frankfurt: Editorial A M Suhrkamp, 1980.

10. SSA, Norma Oficial Mexicana NOM-190-SSA-1999. Prestación de servicios de salud. Criterios para la atención de la violencia familiar, INSP/ SSA, 1999.

I I. INSP/SSA. Encuesta Nacional sobre Violencia contra las Mujeres 2003. México: INSP/SSA, 2003.

12. Glaser B, Strauss A. The discovery of grounded theory. strategies for qualitative research. Chicago: Aldine, 1967.

13. Strauss A, Corbin J. Basics of qualitative research. Newbury Park: Sage, 1990. 